\title{
Diagnostic accuracy of cardiac magnetic resonance tissue tracking technology for differentiating between acute and chronic myocardial infarction
}

\author{
Huaibi Huo ${ }^{1 \#}$, Xu Dai ${ }^{1 \#}$, Simiao Li ${ }^{1}$, Yue Zheng ${ }^{1}$, Jie Zhou ${ }^{1}$, Yao Song ${ }^{1}$, Shuang Liu ${ }^{2}$, Yang Hou ${ }^{3}$, Ting Liu ${ }^{1}$ \\ ${ }^{1}$ Department of Radiology, The First Affiliated Hospital of China Medical University, Shenyang, China; ${ }^{2}$ Department of Ultrasound, The First \\ Affiliated Hospital of China Medical University, Shenyang, China; ${ }^{3}$ Department of Radiology, Shengiing Hospital of China Medical University, \\ Shenyang, China
}

\#These authors contributed equally to this work.

Correspondence to: Ting Liu, MD. Department of Radiology, First Affiliated Hospital of China Medical University, 155 Nanjing Bei Street, Heping, Shenyang 110001, China. Email: cmuliuting@sina.cn.

Background: This study aimed to explore the diagnostic accuracy of cardiac magnetic resonance tissue tracking (CMR-TT) technology in the quantitative evaluation of left myocardial infarction for differentiating between acute and chronic myocardial infarction.

Methods: A total of 104 human subjects were enrolled in this prospective study. Among them, 64 healthy subjects and 40 patients with left ventricular myocardial infarction and 7 days and 6 months' follow-up CMR studies, including steady-state free precession (SSFP) sequence and late gadolinium enhancement MR imaging, were enrolled. The strain parameters of the infarcted myocardium, its corresponding remote segments, and global right ventricular strain were analyzed using tissue tracking technology, and CMR-TT 3D strain parameters in radial, circumferential, and longitudinal directions were obtained. Receiver operating characteristic (ROC) analysis was used to determine the diagnostic accuracy of the CMRTT strain parameters for discriminating between acute and chronic myocardial infarction.

Results: Peak radial strain (RS) of infarcted myocardium increased from $12.99 \pm 7.28$ to $18.57 \pm 6.66$ at 6 months $(\mathrm{P}<0.001)$, whereas peak circumferential strain $(\mathrm{CS})$ increased from $-8.82 \pm 4.71$ to $-12.78 \pm 3.55$ $(\mathrm{P}<0.001)$. CS yielded the best areas under the ROC curve (AUC) of 0.751 in showing differentiation between acute and chronic myocardial infarction of all the strain parameters obtained. The highest significant differences between acute myocardial infarction and normal myocardium, both in the left and right ventricles, were also found in the $\mathrm{RS}(\mathrm{P}<0.001)$ and $\mathrm{CS}(\mathrm{P}<0.001)$.

Conclusions: RS and CS obtained by CMR-TT have high sensitivity and specificity in the differential diagnosis of acute versus chronic myocardial infarction, and their use is thus worth popularizing in clinical application.

Keywords: Cardiac magnetic resonance (CMR); tissue tracking; acute myocardial infarction (AMI); chronic myocardial infarction (CMI); myocardial strain

Submitted Sep 29, 2020. Accepted for publication Feb 24, 2021.

doi: 10.21037 /qims-20-1109

View this article at: http://dx.doi.org/10.21037/qims-20-1109 


\section{Introduction}

Myocardial infarction (MI) is characterized by myocyte cell death, usually precipitated by a profound reduction in blood flow to a region of the heart muscle followed by the development of replacement fibrosis at the injury site. Although the clinical laboratory results after MI are heterogeneous, it is difficult to distinguish between acute myocardial infarction (AMI) and chronic myocardial infarction (CMI). Furthermore, the examination and followup treatment to AMI and CMI are quite different. Although late gadolinium enhancement sequence (LGE) has been demonstrated as a gold standard method to evaluate infarct size and myocardium infarction regions (1), it is of limited value for distinguishing between $\mathrm{AMI}$ and $\mathrm{CMI}$.

In recent years, cardiac magnetic resonance tissue tracking (CMR-TT) technology has been widely used in various heart diseases' clinical research and practice. As a noninvasive technology without contrast, CMR-TT can quantitatively evaluate the early deformation of the left and right myocardium (2). Measurements of myocardial strain and the strain rate can objectively reflect the changes of ventricular structure and function after MI $(3,4)$ and may be able to distinguish between the acute and chronic stages. Therefore, the purpose of this study was to explore the diagnostic accuracy of the quantitative assessment of left myocardial infarction in differentiating between AMI and CMI using CMR-TT.

\section{Methods}

\section{Study cobort}

The institutional ethics committee approved the study, and all subjects gave written informed consent. Patients with a first reperfused ST-segment elevation myocardial infarction (STEMI) were included in the study. Next, 74 recruited patients were screened for study participation, and 40 patients with left ventricular acute myocardial infarction (LVAMI) and 6 months' follow-up were prospectively enrolled between February 2018 and October 2018. Follow-up of these patients was carried out after 6 months. The inclusion criteria were those of established diagnostic criteria (5), while patients with right ventricular myocardial infarction were excluded. Patients were treated with reperfusion therapy utilizing primary percutaneous coronary intervention (PCI). Adequate restoration of blood flow of the infarct-related artery was determined on coronary angiograms by 2 experienced observers with at least 5 years of experience who interpreted coronary angiograms according to the Thrombolysis in Myocardial Infarction (TIMI) trial criteria (6). Study exclusion criteria were as follows: no informed consent $(\mathrm{n}=13)$, participation in a pharmaceutical study $(\mathrm{n}=10)$, hemodynamically unstable at baseline $(n=3)$, severe obesity $(n=2)$, and presence of intracranial metal $(\mathrm{n}=0)$ (Figure 1). For controls, 64 healthy subjects (mean age $55 \pm 9$ years; range $39-71$ years; $69 \%$ male) without any cardiovascular disease symptoms and with normal electrocardiogram (ECG) results were included.

\section{CMR acquisition and image analysis}

Cardiac magnetic resonance imaging (MRI) studies were performed at 7 days and 6 months post PCI on a $3.0 \mathrm{~T}$ system (MAGNETOM Verio, Siemens Healthcare, Erlangen, Germany). Short-axis balanced steady-state free precession (SSFP), cine imaging, and late gadolinium enhancement (LGE) covering the entire heart were performed. Typical imaging parameters for cine-SSFP were repetition time $/$ time to echo $(\mathrm{TR} / \mathrm{TE})=40.4 / 2.4 \mathrm{~ms}$, flip angle $=12^{\circ}$, and GRAPPA (generalized autocalibrating partial parallel acquisition) with a parallel acceleration factor of 2 . The typically used late gadolinium enhancement (LGE) imaging parameters were TR/TE $=750 \mathrm{~ms} / 1.6 \mathrm{~ms}$, inversion time $(\mathrm{TI})=300 \mathrm{~ms}$, flip angle $=20^{\circ}$, and bandwidth $=465 \mathrm{~Hz} /$ pixel. Phase-sensitive inversion recovery (PSIR) was obtained after 8-10 minutes of gadoliniumdiethylenetriamine penta-acetic acid (Gd-DTPA) injection.

Following this, the acquired MR images were processed by CVI42 (version 5.3.4, Circle Cardiovascular Imaging, Canada) by 2 experienced radiologists with more than 6 years of CMR experience (HH and SL). The tissue tracking analysis was performed by drawing out the long axis and the short axis of the endo- and epicardial border. The trabecula was carefully excluded, and the mitral and tricuspid plane and the apex were marked on the long axis and the short axis of the left ventricle (LV) and right ventricle (RV). The endocardium and epicardium were drawn on the maximum diastolic phase cine images at each short-axis level and at the 4-chamber view and left ventricle outflow tract (LVOT) level. The software automatically tracked the displacement of the endocardium and epicardium to quantify the left and right ventricular myocardium movement and divided the ventricle into a basal segment, middle segment, and apical segment.

Additionally, 17 myocardial segments were obtained according to the 2002 American Heart Association (7) guidelines, respectively showing each segment's myocardial 


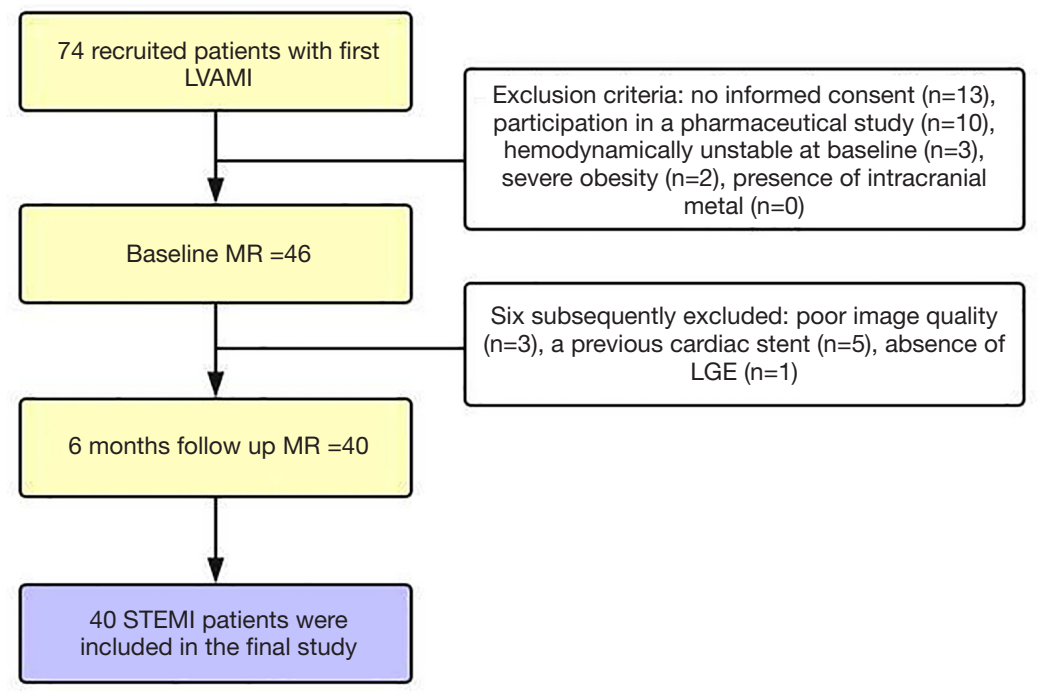

Figure 1 Patient flowchart. The exclusion criteria and patients who did not undergo follow-up are indicated. LVAMI, left ventricular acute myocardial infarction; LGE, late gadolinium enhancement sequence; STEMI, ST-segment elevation myocardial infarction.

strain and strain rate (Figure 2). A consensus was reached between readers in cases of disagreement. LGE was taken as the golden standard to identify infarcted segments (1) and corresponding remote normal segments from the 17-segment image. Myocardial infarctions (MIs) were identified on LGE images and quantitated using a threshold set at 5 standard deviations (SDs) above the remote myocardium's mean signal intensity. Finally, regions of microvascular obstruction (MVO) were manually delineated as those parts of the MI zone that were hypointense.

\section{Statistical methods}

SPSS version 25 (IBM, Armonk, NY, USA) was used to conduct statistical analyses. Measurement data are expressed as mean $\pm \mathrm{SD}$. Independent samples $t$-test and receiver operating characteristic (ROC) curve were established for the diagnosing values of global and regional strain parameters in the LV and RV. Data between infarcted and remote normal myocardium in different stages were compared by using 2 -sided paired $t$-tests; a $\mathrm{P}$ value $<0.05$ indicated a statistically significant difference.

\section{Results}

\section{Patient characteristics}

Initially, 74 patients were recruited, with 6 subsequently excluded due to poor image quality $(\mathrm{n}=3)$, a previous cardiac stent $(n=5)$, and absence of LGE $(n=1)$. In the final study cohort, 40 STEMI patients with LVAMI and 6 months' follow-up MRI were included (Figure 1). The basic characteristics of the 40 patients (mean age $55 \pm$ 7 years; range 34-66 years; $85 \%$ male) with $L V$ myocardial infarction and the 64 healthy subjects (mean age $55 \pm$ 9 years; range $39-71$ years; $69 \%$ male) who underwent CMR scanning are shown in Table 1, while the patients' clinical features are shown in Table 2.

\section{Evaluation of strain parameters between the infarcted and remote myocardia}

The strain parameters of peak radial strain (RS), peak circumferential strain (CS), and peak radial systolic strain rate (RS-s') $(\mathrm{P}<0.001$ for all) of infarcted myocardia at baseline were significantly lower than those of remote myocardia, while CS showed the greatest differentiation ability with the largest area under the ROC curve (AUC) of 0.895 (Figure 3). When a CS of $<-14.47$ was used as the AMI diagnostic area, the sensitivity and specificity were $95 \%$ and $75 \%$, respectively. RS ( $\mathrm{P}=0.001)$, CS $(\mathrm{P}=0.001)$, and RS-s' $(\mathrm{P}=0.003)$ of infarcted myocardia remained lower than those of remote myocardia and progressively increased over the course of 6 months (Table 3, Figure 4). We further found significant differences in longitudinal strain (LS), peak circumferential systolic strain rate (CS-s'), and peak 

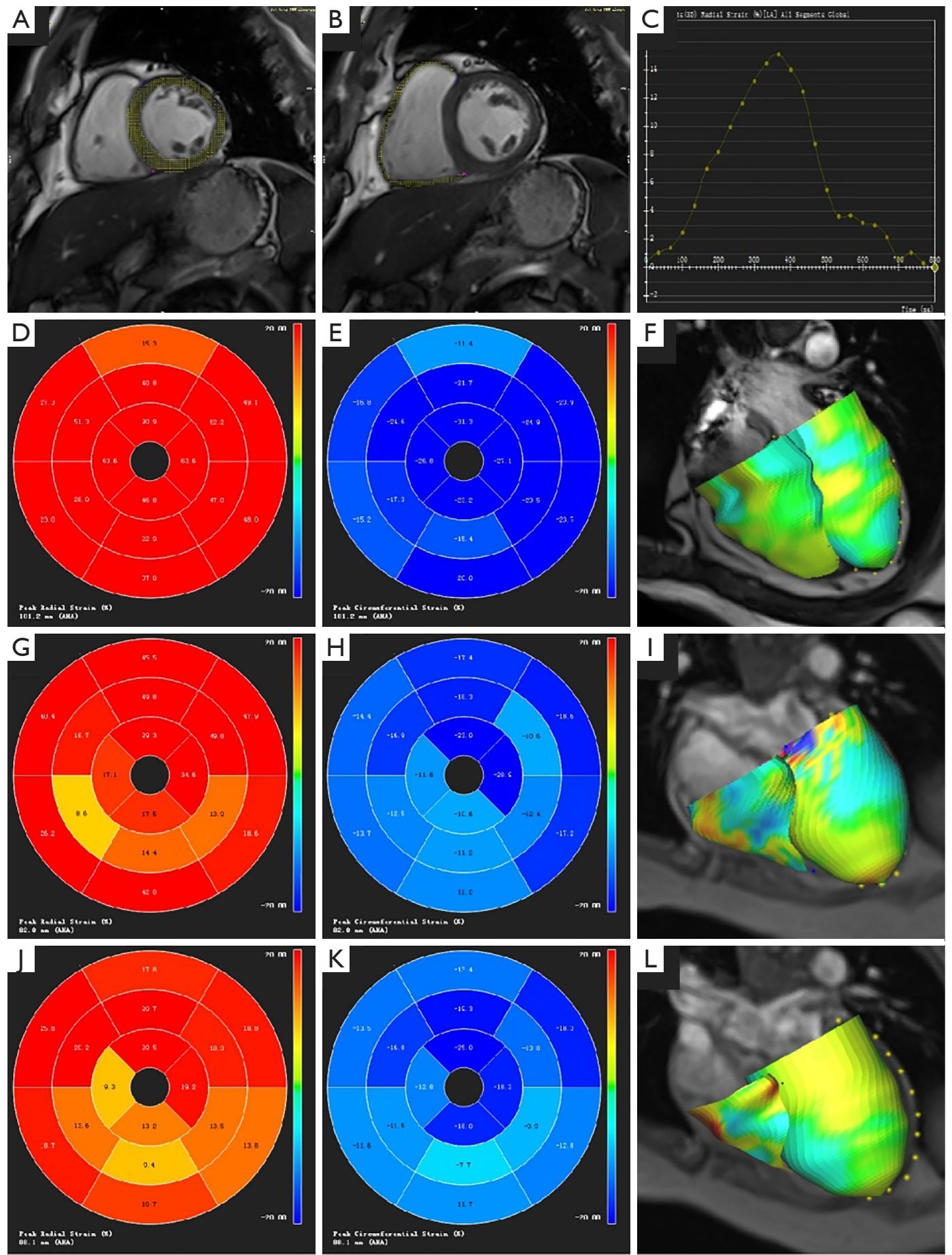

Figure 2 The CMR-TT. (A,B,C) Short-axis circumferential strain. The yellow represents the tracking for the ventricle. (A) Left ventricle (LV); (B) right ventricle (RV). The images are all diastolic images. (C) The curve showing the radial strain values of the LV in 25 phases, including the peak value. (D,E,F) A healthy, 42-year-old male. (G,H,I) A 54-year-old female, with acute myocardial infarction (AMI) at baseline (J,K,L) and 6 months after AMI. (D,G,J) The polar map of 2002 American Heart Association segmentation for radial strain. (E,H,K) Images illustrating the circumferential strain. (F,I,L) 3D models of $\mathrm{LV}$ and RV in diastole; the color coding represents the epicardial contours. CMR-TT, cardiac magnetic resonance tissue tracking. 
Table 1 Patient characteristics

\begin{tabular}{|c|c|c|c|c|c|}
\hline Variables & MI patients (baseline, $n=40$ ) & MI patients ( 6 months $F U, n=40$ ) & Healthy subjects $(n=64)$ & $P_{1}$ values & $\mathrm{P}_{2}$ values \\
\hline Height $(\mathrm{cm})$ & $170.7 \pm 6.6$ & $170.7 \pm 6.6$ & $168.8 \pm 6.8$ & 0.274 & 0.274 \\
\hline Weight (kg) & $65.1 \pm 10.3$ & $65.0 \pm 10.5$ & $64.9 \pm 10.4$ & 0.898 & 0.997 \\
\hline $\mathrm{BMI}\left(\mathrm{kg} / \mathrm{m}^{2}\right)$ & $22.2 \pm 2.3$ & $22.2 \pm 2.4$ & $22.6 \pm 2.1$ & 0.888 & 0.951 \\
\hline LVEDVi $\left(\mathrm{mL} / \mathrm{m}^{2}\right)$ & $73.5 \pm 16.6$ & $72.8 \pm 19.2$ & $55.8 \pm 7.9$ & 0.000 & 0.000 \\
\hline RVEF (\%) & $48.6 \pm 6.8$ & $48.9 \pm 5.9$ & $49.7 \pm 7.4$ & 0.655 & 0.079 \\
\hline RVEDVi $\left(\mathrm{mL} / \mathrm{m}^{2}\right)$ & $49.4 \pm 8.7$ & $51.0 \pm 10.6$ & $58.3 \pm 11.5$ & 0.019 & 0.507 \\
\hline
\end{tabular}

There was no significant difference between baseline and 6-month follow-up. $P_{1}$ for baseline versus healthy subjects. $P_{2}$ for 6-month follow-up versus healthy controls. MI, myocardial infarction; FU, follow-up; LV, left ventricle; RV, right ventricle; EF, ejection fraction; EDVi, end-diastolic volume index.

Table 2 Patient clinical features

\begin{tabular}{|c|c|c|c|}
\hline Variables & MI patients (baseline, $n=40$ ) & MI patients ( 6 months $\mathrm{FU}, \mathrm{n}=40$ ) & $P$ values \\
\hline \multicolumn{4}{|l|}{ Infarct-related artery } \\
\hline Left anterior descending & $34(85 \%)$ & $34(85 \%)$ & - \\
\hline Left circumflex & $6(15 \%)$ & $6(15 \%)$ & - \\
\hline \multicolumn{4}{|c|}{ Cardiac magnetic resonance findings } \\
\hline Infarct volume (\%LV) & $35.81 \pm 15.22$ & $34.38 \pm 13.03$ & 0.746 \\
\hline MVO volume (\%LV) & $3.38 \pm 3.09$ & $3.00 \pm 2.00$ & 0.638 \\
\hline
\end{tabular}

MI, myocardial infarction; FU, follow-up; MVO, microvascular obstruction.

longitudinal systolic strain rate (LS-s') between infarcted and remote myocardia in LVAMI patients $(\mathrm{P}=0.015$, $\mathrm{P}=0.007$, and $\mathrm{P}=0.010$, respectively), but no significant differences in LVCMI patients were observed $(\mathrm{P}=0.081$, $\mathrm{P}=0.526$, and $\mathrm{P}=0.338$, respectively; Table 3).

\section{Cardiac MR strain parameters for differentiating between $A M I$ and $C M I$}

There were significant differences in the parameters of RS, CS, RS-s', and CS-s' between AMI and CMI $(\mathrm{P}<0.05$ for all), but no significant difference in LS ( $\mathrm{P}=0.791)$ and LS-s' $(\mathrm{P}=0.361$; Table 3). The RS of infarcted myocardia increased from $12.99 \pm 7.28$ to $18.57 \pm 6.66$ at 6 months $(\mathrm{P}<0.001)$ CS increased from $-8.82 \pm 4.71$ to $-12.78 \pm 3.55(\mathrm{P}<0.001)$, demonstrating the largest change. Of all the CMR-TT strain parameters obtained, CS yielded the best AUC (Figure 3) of 0.751 for differentiating between AMI and CMI. When CS $<-14.57$ was taken as the boundary point of diagnosing AMI or CMI, the sensitivity and specificity were $55 \%$ and $95 \%$, respectively. We also explored the global and regional CMR-TT strain parameters of LV in healthy participants and LVMI patients. The comparative analysis results are shown in Table 4. The strain parameters of global and regional RS, CS, and LS ( $\mathrm{P}<0.001$ for all) of infarcted myocardia at both baseline and 6 months were significantly lower than those of remote myocardia.

\section{Evaluation of $R V$ strain parameters in LVMI patients}

We explored the global and regional CMR-TT strain parameters of RV in healthy participants versus LVMI 
A

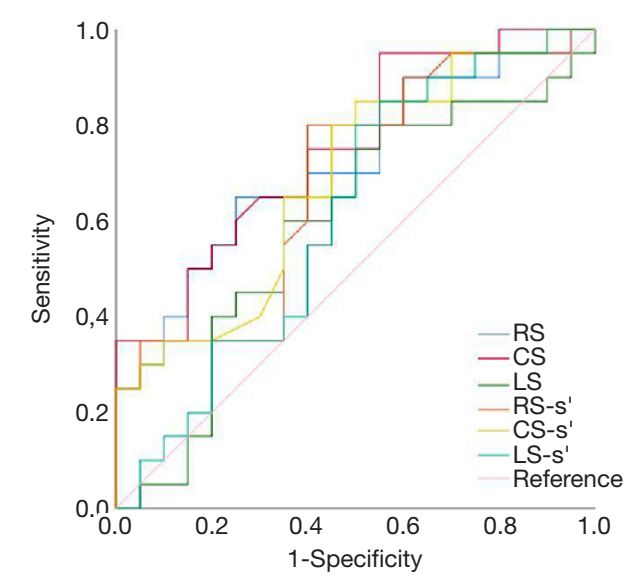

C

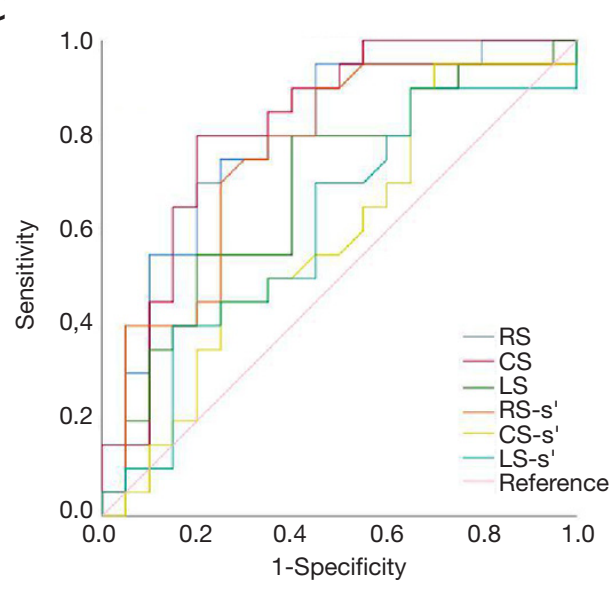

B

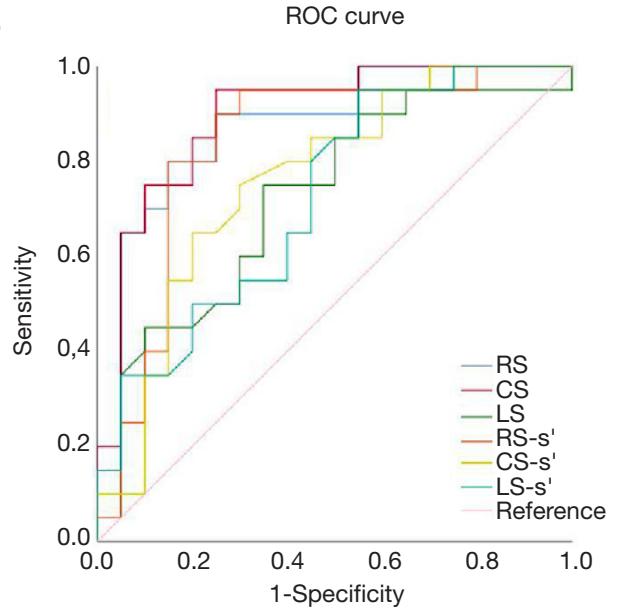

Figure 3 Graphs showing results of ROC analysis for differentiation between AMI and CMI. (A) The results of ROC analysis for differentiating the infarct myocardia of AMI and CMI (the AUCs of RS, CS, LS, RS-s', CS-s', and LS-s' were 0.715, 0.751, 0.590, 0.685, 0.687 , and 0.612 , respectively). (B) ROC analysis for differentiating between the infarcted myocardia and remote normal myocardia in AMI patients (the AUCs of RS, CS, LS, RS-s', CS-s' and LS-s' were $0.878,0.895,0.727,0.835,0.761$, and 0.722 respectively). (C) ROC analysis for differentiating between infarcted myocarda and remote normal myocardia in CMI patients (the AUCs of RS, CS, LS, RS-s', CS-s', and LS-s' were $0.800,0.820,0.690,0.758,0.587$, and 0.609 respectively). AMI, acute myocardial infarction; CMI, chronic myocardial infarction; RS, peak radial strain; CS, peak circumferential strain; LS, peak longitudinal strain; RS-s', peak radial systolic strain rate; CS-s', peak circumferential systolic strain rate; LS-s', peak longitudinal systolic strain rate.

patients. The comparative analysis results are shown in Table 5. The global peak radial strain (GRS), global peak circumferential strains (GCS), and global peak longitudinal strain (GLS) of the RV in the healthy participants were $25.75 \pm 6.28,-15.54 \pm 2.52$, and $-26.74 \pm 5.56$, respectively. There were significant differences in GRS $(\mathrm{P}<0.001)$, GCS $(\mathrm{P}<0.001)$, GLS $(\mathrm{P}=0.001)$, peak radial systolic strain rate (GRS-s' $\mathrm{P}=0.003)$, and baseline GRS $(\mathrm{P}<0.001)$ and GCS $(\mathrm{P}<0.001)$, with greater reductions shown in AMI patients as compared to controls. At a 6-month follow-up, the GCS
$(\mathrm{P}=0.009)$, GLS $(\mathrm{P}=0.007)$, and global peak circumferential strain for basal segment (GCS-b) $(\mathrm{P}<0.001)$ of LVMI patients remained lower than those of controls; however, there was no significant difference in GRS $(\mathrm{P}=0.086)$ or GRS-s' (P=0.061).

\section{Discussion}

The current study evaluated the diagnostic accuracy of strain parameters by CMR-TT for differentiating between 
Table 3 Strain values of the left ventricle in infarcted and remote normal myocardia at baseline and 6 months later

\begin{tabular}{|c|c|c|c|c|c|c|c|c|}
\hline $\begin{array}{l}\text { MR imaging } \\
\text { parameter }\end{array}$ & \multicolumn{2}{|c|}{ Infarcted } & \multicolumn{2}{|c|}{ Remote } & $\mathrm{P}_{1}$ values & $\mathrm{P}_{2}$ values & $\mathrm{P}_{3}$ values & $\mathrm{P}_{4}$ values \\
\hline RS & $12.99 \pm 7.28$ & $18.57 \pm 6.66$ & $27.46 \pm 9.71$ & $27.38 \pm 8.12$ & 0.000 & 0.963 & 0.000 & 0.001 \\
\hline CS & $-8.82 \pm 4.71$ & $-12.78 \pm 3.55$ & $-16.52 \pm 4.28$ & $-17.05 \pm 3.58$ & 0.000 & 0.538 & 0.000 & 0.001 \\
\hline LS & $-7.61 \pm 7.11$ & $-8.29 \pm 8.02$ & $-13.16 \pm 6.65$ & $-12.75 \pm 7.69$ & 0.791 & 0.823 & 0.015 & 0.081 \\
\hline CS-s' & $-0.52 \pm 0.39$ & $-0.80 \pm 0.38$ & $-0.88 \pm 0.41$ & $-0.88 \pm 0.40$ & 0.004 & 0.939 & 0.007 & 0.526 \\
\hline LS-s' & $-0.26 \pm 0.75$ & $-0.51 \pm 1.14$ & $-0.90 \pm 0.73$ & $-0.83 \pm 0.21$ & 0.361 & 0.788 & 0.010 & 0.338 \\
\hline
\end{tabular}

$P_{1}$ for baseline versus 6 months in infarcted myocardia. $P_{2}$ for baseline versus 6 months in remote normal myocardia. $P_{3}$ for infarcted myocardia versus remote normal myocardia at baseline. $P_{4}$ for infarcted myocardia versus remote normal myocardia at 6 months. RS, peak radial strain; CS, peak circumferential strain; LS, peak longitudinal strain; RS-s', peak radial systolic strain rate; CS-s', peak circumferential systolic strain rate; LS-s', peak longitudinal systolic strain rate.
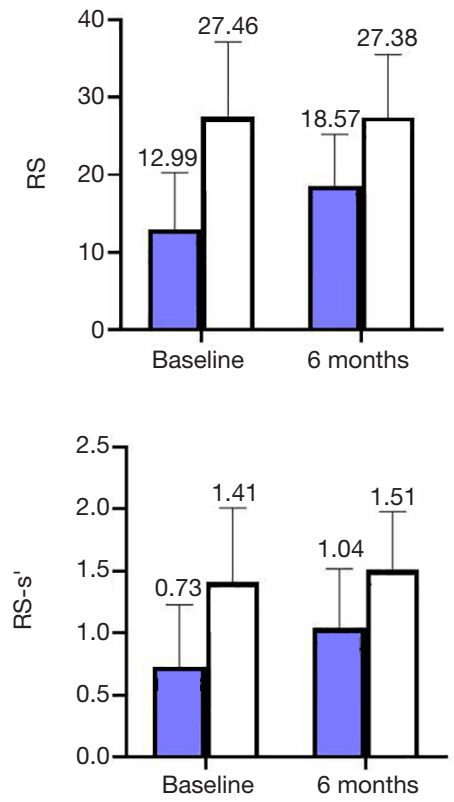
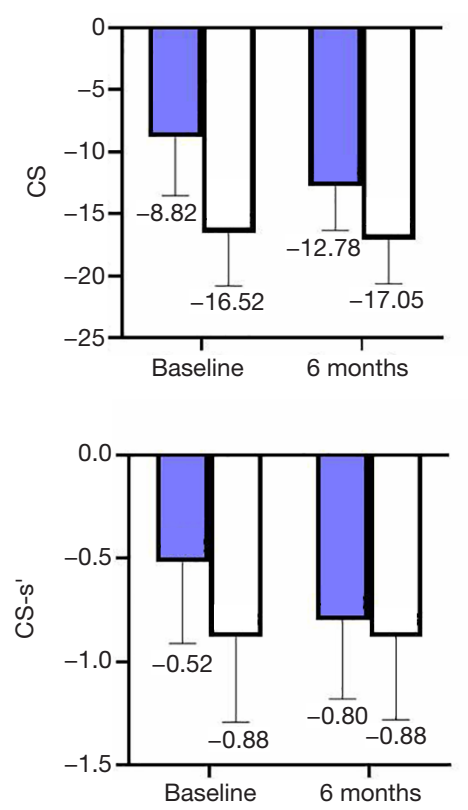
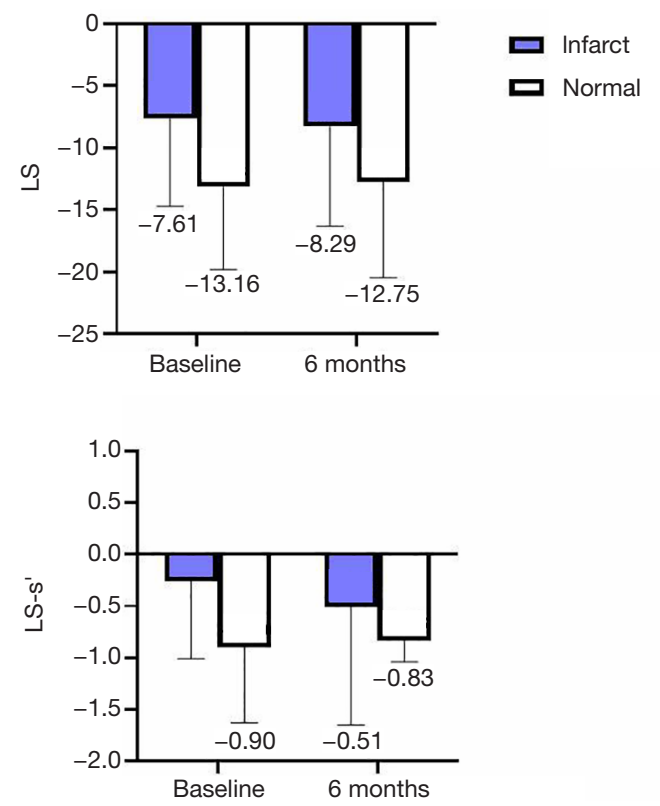

Figure 4 Bar graphs showing the development of RS, CS, LS, RS-s', CS-s', and LS-s' after AMI in infarcted and remote normal myocardia. RS and RS-s' showed a progressive increase while CS and CS-s' showed a progressive decline in infarcted myocardium within the first 6 months after AMI $\left(\mathrm{P}_{1}<0.05\right)$. All the parameters in the remote normal myocardia showed minimal change between the acute and chronic stages $\left(\mathrm{P}_{2}>0.05\right)$. AMI, acute myocardial infarction; RS, peak radial strain; CS, peak circumferential strain; LS, peak longitudinal strain; RSs', peak radial systolic strain rate; CS-s', peak circumferential systolic strain rate; LS-s', peak longitudinal systolic strain rate.

AMI and CMI. Our findings provide relevant proof of concept for the role of quantitative tissue techniques in noncontrast methods for recognizing and monitoring the staging of myocardial infarction in MI patients. Based on the parallel relationship between LV and RV strain measurement, LV deformation and edema are the predominant drivers for differentiating between AMI and CMI. The results of our study provide several important advances: (I) CS and RS, but not LS, progressively increased within the first 6 months after infarction; (II) cardiac MR strain analysis could be used for monitoring the staging of myocardial infarction in MI patients; (III) CS of infarcted 
Table 4 Global strain values of left ventricular myocardia at baseline and 6 months later in LVMI patients and normal controls

\begin{tabular}{lccccc}
\hline Variables & Baseline (acute) & 6 months (chronic) & Normal & $P_{1}$ values & $P_{2}$ values \\
\hline GRS & $18.90 \pm 5.96$ & $21.72 \pm 5.84$ & $45.29 \pm 9.78$ & 0.139 & 0.000 \\
GCS & $-12.61 \pm 3.14$ & $-14.09 \pm 2.98$ & $-21.90 \pm 2.85$ & 0.134 & 0.000 \\
GLS & $-10.15 \pm 3.41$ & $-12.15 \pm 2.56$ & $-19.37 \pm 2.47$ & 0.043 & 0.000 \\
GRS-b & $26.36 \pm 7.05$ & $27.40 \pm 5.42$ & $44.34 \pm 9.51$ & 0.604 & 0.000 \\
GRS-m & $18.77 \pm 6.71$ & $21.48 \pm 6.63$ & $44.54 \pm 12.03$ & 0.208 & 0.000 \\
GRS-a & $13.91 \pm 8.36$ & $18.62 \pm 10.13$ & $53.84 \pm 15.08$ & 0.117 & 0.000 \\
GCS-b & $-16.06 \pm 2.95$ & $-16.47 \pm 2.27$ & $-20.71 \pm 2.82$ & 0.621 & 0.000 \\
GCS-m & $-12.75 \pm 3.66$ & $-14.23 \pm 3.15$ & $-21.94 \pm 3.38$ & 0.179 & 0.000 \\
GCS-a & $-9.65 \pm 4.83$ & $-12.33 \pm 5.30$ & $-24.11 \pm 3.58$ & 0.103 & 0.000 \\
GRS-s' & $0.93 \pm 0.24$ & $1.02 \pm 0.29$ & $2.39 \pm 0.61$ & 0.000 & 0.000 \\
GCS-s' & $-0.64 \pm 0.13$ & $-0.69 \pm 0.14$ & $-1.09 \pm 0.22$ & 0.000 & 0.000 \\
GLS-s' & $-0.53 \pm 0.30$ & $-0.70 \pm 0.21$ & $-0.96 \pm 0.51$ & 0.053 & 0.000 \\
\hline
\end{tabular}

$\mathrm{P}_{1}$ for baseline versus 6 months strain values in left ventricular myocardia. $\mathrm{P}_{2}$ for LV global strain values at baseline versus normal controls. $P_{3}$ for LV global strain values at 6 months versus normal controls. GRS, global peak radial strain; GCS, global peak circumferential strain; GLS, global peak longitudinal strain; GRS-b, global peak radial strain for basal; GRS-m, global peak radial strain for mid segment; GRS-a, global peak radial strain for apical segment; GCS-b, global peak circumferential strain for basal segment; GCS-m, global peak circumferential strain for mid segment; GCS-a, global peak circumferential strain for apical segment; GRS-s', peak radial systolic strain rate; GCS-s', peak circumferential systolic strain rate; GLS-s', peak longitudinal systolic strain rate.

Table 5 Global strain values of right ventricular myocardia at baseline and 6 months later in LVMI patients and normal controls

\begin{tabular}{|c|c|c|c|c|c|c|}
\hline Variables & Baseline (acute) & 6 months (chronic) & Normal & $P_{1}$ values & $P_{2}$ values & $P_{3}$ values \\
\hline GCS & $-11.73 \pm 2.67$ & $-13.19 \pm 3.74$ & $-15.54 \pm 2.52$ & 0.021 & 0.000 & 0.009 \\
\hline GLS & $-20.76 \pm 6.68$ & $-22.46 \pm 5.01$ & $-26.74 \pm 5.56$ & 0.273 & 0.001 & 0.007 \\
\hline GRS-b & $14.09 \pm 8.22$ & $16.65 \pm 13.05$ & $25.12 \pm 10.93$ & 0.377 & 0.000 & 0.015 \\
\hline GRS-a & $27.48 \pm 9.88$ & $28.79 \pm 10.82$ & $34.95 \pm 11.82$ & 0.609 & 0.022 & 0.065 \\
\hline GCS-b & $-5.25 \pm 8.42$ & $-6.66 \pm 9.61$ & $-14.69 \pm 4.73$ & 0.462 & 0.000 & 0.000 \\
\hline GCS-m & $-12.90 \pm 3.31$ & $-15.16 \pm 3.10$ & $-14.51 \pm 3.00$ & 0.007 & 0.077 & 0.458 \\
\hline GCS-a & $-16.20 \pm 4.08$ & $-16.58 \pm 4.46$ & $-19.00 \pm 4.15$ & 0.757 & 0.021 & 0.053 \\
\hline GLS-s' & $-0.89 \pm 1.27$ & $-1.01 \pm 0.92$ & $-1.10 \pm 1.64$ & 0.690 & 0.627 & 0.833 \\
\hline
\end{tabular}

$P_{1}$ for baseline versus 6 months strain values in right ventricular myocardia. $P_{2}$ for right ventricular global strain values at baseline versus normal controls. $P_{3}$ for right ventricular global strain values at 6 months versus normal controls. GRS, global peak radial strain; GCS, global peak circumferential strain; GLS, global peak longitudinal strain; GRS-b, global peak radial strain for basal segment; GRS-m, global peak radial strain for mid segment; GRS-a, global peak radial strain for apical segment; GCS-b, global peak circumferential strain for basal segment; GCS-m, global peak circumferential strain for mid segment; GCS-a, global peak circumferential strain for apical segment; GRS-s', peak radial systolic strain rate; GCS-s', peak circumferential systolic strain rate; GLS-s', peak longitudinal systolic strain rate. 
myocardium was the best at differentiating between AMI and CMI, with high diagnostic accuracy in all patients; (IV) there were significant differences in the RV GRS, GCS, and GLS between AMI, CMI, and controls. Thus, the quantitative measurement of infarcted myocardia's strain parameters may serve as a useful metric for noninvasive evaluation, detection, and discrimination of acute and chronic infarction.

Myocardial infarction caused by $\mathrm{LV}$ coronary heart disease is a disease related to myocardial ischemia. Myocardial ischemia can lead to myocardial deformation in the lesion segment, the decline of myocardial function, and a decrease in exercise-these changes of the myocardium resulting in the involvement of the endocardium and epicardium. The contraction of endocardial fibers results in the longitudinal shortening of the myocardium related to LS and LS-s'. Contraction of epicardial fibers results in the circumferential shortening of the myocardium, which is related to CS and CS-s'; this then results in the myocardium's radial thickening, leading to a change in RS and RS-s' (8). In the early stage of MI, the endocardial myocardium-as a remote blood supply area-becomes ischemic earlier than the epicardial myocardium. The LS decreases, while the RS and CS compensate (8) to maintain the normal cardiac function. Thus, myocardial stress can reflect a myocardial abnormality earlier than the decrease of ejection fraction (EF), as has already been confirmed by several studies (9-12).

Furthermore, the strain value, which has been suggested as a potential tool for evaluating early or subclinical changes of $L V$ function, performs better than LVEF in reflecting myocardial deformation and has already been incorporated into alternative measurements of therapeutic results $(13,14)$. Many clinical studies (15), including meta-analyses, have confirmed that timely vascular reperfusion therapy can reduce AMI patients' annual mortality. The reason for this lies in the presence of the surviving myocardium. Therefore, distinguishing between AMI and CMI has clinical value and can effectively improve the prognosis and survival rate.

In the acute MI examined in our study, the absolute values of RS, CS, LS, RS-s', CS-s,' and LS-s' in the infarcted segments were significantly lower than those in the distal normal areas (Figure 4), with this difference being statistically significant, especially for RS and CS $(\mathrm{P}<0.001)$. In this stage, the myocardial function is affected by ischemia and necrosis of myocardial infarction segments, activation of the inflammatory response, and phagocytosis of necrotic cardiomyocytes $(16,17)$. Therefore, the myocardial function of infarcted segments decreases rapidly, and the coordinated contractile force of the myocardium decreases, rendering it unable to produce effective contractions. The compensatory enhancement of myocardial function can maintain the effective ejection function of LV (15). Neizel et al. (18) used strain-encoded imaging (SENC) MRI in 38 patients with AMI to compare transmural infarcted and noninfarcted segments to identify a threshold for CS of $-10 \%$, with a corresponding sensitivity of $97 \%$ and a specificity of $94 \%$. A similar study performed by McComb et al. (19) found that a CS of $-12.8 \%$ had moderately high values between infarcted and remote segments in 50 male AMI subjects, with an AUC of 0.88 , a sensitivity of $84 \%$, and a specificity of $78 \%$. Our study shows that when a CS $<-14.47$ is used as the inflection point, AMI's diagnostic efficiency is the best, with a corresponding sensitivity of $95 \%$, a specificity of $75 \%$, and an AUC of 0.90. Our findings are consistent with those of several other studies, in that the reductions in CS (18-24), RS $(23,25)$, LS $(18,22,23)$, and CS-s' (26) can discriminate between the infarcted area and normal remote segments in AMI patients, with CS showing better discrimination ability than LS (20). Due to significant variations in strain values obtained by different software solutions and measurement techniques, these parameters should be quantified similarly if serial assessments or follow-up acquisitions are required; otherwise, the threshold identification may be affected.

In chronic MI, the absolute values of RS, CS, RS-s', and CS-s' in the infarcted segments were significantly higher than those in the same lesion in the acute MI, towarding the strain level of normal myocardial segments (Figure 4), with these differences being statistically significant $(\mathrm{P}<0.05)$. The absolute values of RS, CS, and RS-s' in the chronic myocardial infarction area were still significantly lower than those in the remote normal area, with these differences being statistically significant $(\mathrm{P}<0.05)$. The results showed that with the prolongation of time, the infarcted myocardium could be remolded by the inflammatory reaction to form a fibrous scar. Although the deformation of the infarcted segment was still changed to some extent, it had not changed much, which indicates that the remodeling and deformation of the LV tended to be stable at the moment, that the myocardial load was reduced, and that, simultaneously, the RS, CS, and RS-s' of infarcted segments changed significantly $(15,16,27)$. In our study, LS and LSs' showed a significant reduction in AMI patients but no significant changes in CMI patients (Table 3), indicating that LS can, to some extent, recover after a certain period.

Our study also obtained the myocardial strain parameters of the RV. Liu et al. (28) reported the normal reference of 
A

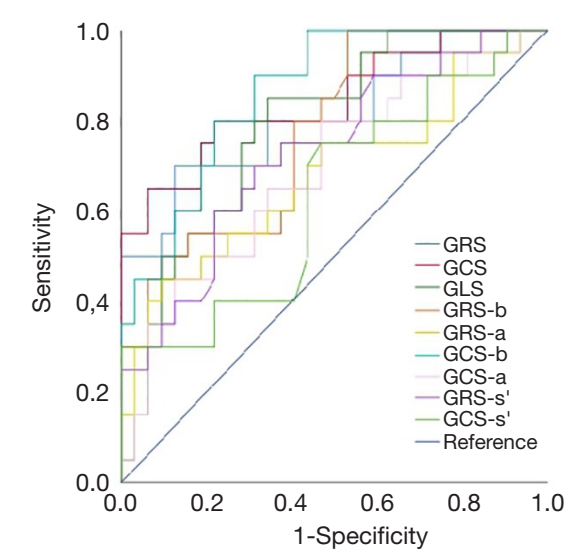

B

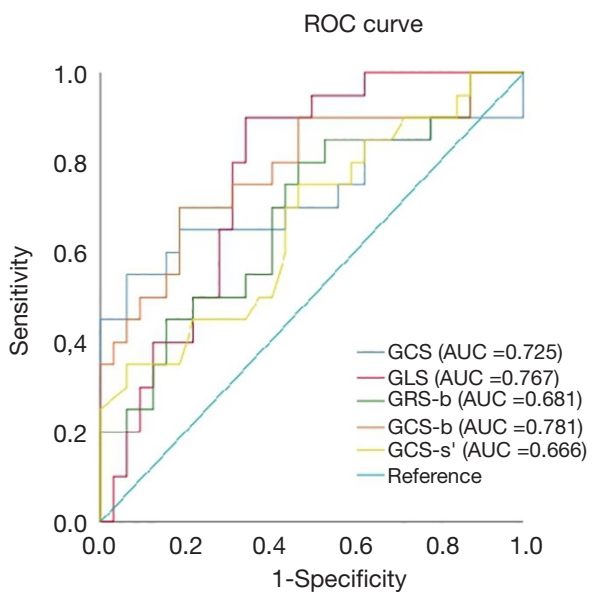

Figure 5 Graphs showing results of ROC analysis for differentiation in right ventricular myocardia between (A) LVAMI and normal controls (the AUCs of GRS, GCS, GLS, GRS-b, GRS-a, GCS-b, GCS-a, GRS-s', and GCS-s' were 0.813, 0.844, 0.788, 0.776, 0.680, 0.864, 0.691, 0.73 , and 0.635 , respectively), and (B) between LVCMI and normal controls (the AUCs of GCS, GLS, GRS-b, GCS-b, and GCS-s' were $0.725,0.767,0.681,0.784$, and 0.666 , respectively). LVAMI, left ventricular acute myocardial infarction; LVCMI, left ventricular chronic myocardial infarction; GRS, global peak radial strain; GCS, global peak circumferential strain; GLS, global peak longitudinal strain; GRS-b, global peak radial strain for basal; GRS-a, global peak radial strain for apical; GCS-b, global peak circumferential strain for basal; GCS-a, global peak circumferential strain for apical; GRS-s', peak radial systolic strain rate; GCS-s', peak circumferential systolic strain rate.

RV myocardial strain parameters in 120 normal Chinese volunteers with a GLS of $-24.3 \pm 4.7$, a GRS of $23.0 \pm 8.5$, and a GCS $-13.1 \pm 4.0$. The GLS, GRS, and GCS of our study of 64 healthy subjects were $-26.74 \pm 5.56,25.75 \pm 6.28$, and $-15.54 \pm 2.52$, which were slightly higher. Our data also demonstrated the deformation and remodeling of the RV myocardium in patients with LVMI. GRS, GCS, and mid segments for both strain types could distinguish between acute and chronic MI stages, while basal and apical segments showed no significant difference. This result suggests that myocardial strain at the middle segments involves smaller deformation and better recovery than more obvious deformation and weaker recovery observed at the base and apex. A GCS of $<-13.81$ at the midsegments used as the inflection point showed the best diagnostic efficiency for AMI, with a sensitivity and specificity of $65 \%$ and $65 \%$, respectively (Figure 5). GLS showed a significant difference between LVAMI and LVCMI; however, GLS could discriminate between LVAMI and controls and between LVCMI and controls.

This study's major limitations might be due to its small sample size and the individual variation between the participants. Larger samples would still be needed to determine the diagnostic threshold and promote the quantitative diagnosis between AMI and CMI afforded by this technology.

\section{Conclusions}

In conclusion, RS and CS demonstrated by CMR-TT have higher diagnostic value for differentiating between AMI and CMI and differentiate acute and chronic myocardial infarction segments within the same individual. LS has a degree of diagnostic ability in AMI, but its diagnostic value in $\mathrm{CMI}$ is limited. Overall, our findings suggest that CMR-TT can be employed to reveal infarcted segments at different stages without the use of contrast medium injection.

\section{Acknowledgments}

Funding: This study was funded by the National Nature Science Foundation of China (No. 81871435).

\section{Footnote}

Conflicts of Interest: All authors have completed the ICMJE uniform disclosure form (available at http://dx.doi. org/10.21037/qims-20-1109). The authors have no conflicts of interest to declare. 
Ethical Statement: Ethics Review Board approved this study of the First Affiliated Hospital of China Medical University and all subjects gave written informed consent.

Open Access Statement: This is an Open Access article distributed in accordance with the Creative Commons Attribution-NonCommercial-NoDerivs 4.0 International License (CC BY-NC-ND 4.0), which permits the noncommercial replication and distribution of the article with the strict proviso that no changes or edits are made and the original work is properly cited (including links to both the formal publication through the relevant DOI and the license). See: https://creativecommons.org/licenses/by-nc-nd/4.0/.

\section{References}

1. Carlsson M, Arheden H, Higgins CB, Saeed M. Magnetic resonance imaging as a potential gold standard for infarct quantification. J Electrocardiol 2008;41:614-20.

2. Claus P, Omar AMS, Pedrizzetti G, Sengupta PP, Nagel E. Tissue Tracking Technology for Assessing Cardiac Mechanics: Principles, Normal Values, and Clinical Applications. JACC Cardiovasc Imaging 2015;8:1444-60.

3. Smiseth OA, Torp H, Opdahl A, Haugaa KH, Urheim S. Myocardial strain imaging: how useful is it in clinical decision making? Eur Heart J 2016;37:1196-207.

4. Azak E, Cetin II, Gursu HA, Kibar AE, Surucu M, Orgun A, Pamuk U. Recovery of myocardial mechanics in Kawasaki disease demonstrated by speckle tracking and tissue Doppler methods. Echocardiography 2018;35:380-7.

5. Thygesen K, Alpert JS, Jaffe AS, Chaitman BR, Bax JJ, Morrow DA, White HD; Executive Group on behalf of the Joint European Society of Cardiology (ESC)/American College of Cardiology (ACC)/American Heart Association (AHA)/World Heart Federation (WHF) Task Force for the Universal Definition of Myocardial Infarction. Fourth Universal Definition of Myocardial Infarction (2018). Glob Heart 2018;13:305-38.

6. TIMI Study Group. The Thrombolysis in Myocardial Infarction (TIMI) trial. Phase I findings. N Engl J Med 1985;312:932-6.

7. Cerqueira MD, Weissman NJ, Dilsizian V, Jacobs AK, Kaul S, Laskey WK, Pennell DJ, Rumberger JA, Ryan T, Verani MS; American Heart Association Writing Group on Myocardial Segmentation and Registration for Cardiac Imaging. Standardized myocardial segmentation and nomenclature for tomographic imaging of the heart. A statement for healthcare professionals from the Cardiac Imaging Committee of the Council on Clinical Cardiology of the American Heart Association. Circulation 2002;105:539-42.

8. Sengupta PP, Tajik AJ, Chandrasekaran K, Khandheria BK. Twist mechanics of the left ventricle: principles and application. JACC Cardiovasc Imaging 2008;1:366-76.

9. Hanboly NH, Baghdady YM, Diab RH, Lawend SR, Kenawy AA. Value of three-dimensional echocardiography study of left ventricle function correlated to coronary arterial dominance in predicting the outcome of primary percutaneous coronary intervention. J Saudi Heart Assoc 2018;30:211-21.

10. Bourfiss M, Vigneault DM, Aliyari Ghasebeh M, Murray B, James CA, Tichnell C, Mohamed Hoesein FA, Zimmerman SL, Kamel IR, Calkins H, Tandri H, Velthuis BK, Bluemke DA, Te Riele ASJM. Feature tracking CMR reveals abnormal strain in preclinical arrhythmogenic right ventricular dysplasia/ cardiomyopathy: a multisoftware feasibility and clinical implementation study. J Cardiovasc Magn Reson 2017;19:66.

11. Buckert D, Witzel S, Steinacker JM, Rottbauer W, Bernhardt P. Comparing Cardiac Magnetic ResonanceGuided Versus Angiography-Guided Treatment of Patients With Stable Coronary Artery Disease: Results From a Prospective Randomized Controlled Trial. JACC Cardiovasc Imaging 2018;11:987-96.

12. Andre F, Steen H, Matheis P, Westkott M, Breuninger K, Sander Y, Kammerer R, Galuschky C, Giannitsis E,Korosoglou G, Katus HA, Buss SJ. Age- and genderrelated normal left ventricular deformation assessed by cardiovascular magnetic resonance feature tracking. J Cardiovasc Magn Reson 2015;17:25.

13. Mangion K, McComb C, Auger DA, Epstein FH, Berry C. Magnetic Resonance Imaging of Myocardial Strain After Acute ST-Segment-Elevation Myocardial Infarction: A Systematic Review. Circ Cardiovasc Imaging 2017;10:e006498.

14. Sengupta PP, Narula J. Cardiac strain as a universal biomarker: interpreting the sounds of uneasy heart muscle cells. JACC Cardiovasc Imaging 2014;7:534-6.

15. Sun JP, Niu J, Chou D, Chuang HH, Wang K, Drinko J, Borowski A, Stewart WJ, Thomas JD. Alterations of regional myocardial function in a swine model of myocardial infarction assessed by echocardiographic 2-dimensional strain imaging. J Am Soc Echocardiogr 2007;20:498-504.

16. Mitchell GF, Lamas GA, Vaughan DE, Pfeffer MA. Left 
ventricular remodeling in the year after first anterior myocardial infarction: a quantitative analysis of contractile segment lengths and ventricular shape. J Am Coll Cardiol 1992;19:1136-44.

17. Carlsson E, Milne EN. Permanent implantation of endocardial tantalum screws: a new technique for functional studies of the heart in the experimental animal. J Can Assoc Radiol 1967;18:304-9.

18. Neizel M, Lossnitzer D, Korosoglou G, Schäufele T, Peykarjou H, Steen H, Ocklenburg C, Giannitsis E, Katus HA, Osman NF. Strain-encoded MRI for evaluation of left ventricular function and transmurality in acute myocardial infarction. Circ Cardiovasc Imaging 2009;2:116-22.

19. McComb C, Carrick D, McClure JD, Woodward R, Radjenovic A, Foster JE, Berry C. Assessment of the relationships between myocardial contractility and infarct tissue revealed by serial magnetic resonance imaging in patients with acute myocardial infarction. Int J Cardiovasc Imaging 2015;31:1201-9.

20. Khan JN, Singh A, Nazir SA, Kanagala P, Gershlick AH, McCann GP. Comparison of cardiovascular magnetic resonance feature tracking and tagging for the assessment of left ventricular systolic strain in acute myocardial infarction. Eur J Radiol 2015;84:840-8.

21. Inoue $Y$, Yang $X$, Nagao M, Higashino H, Hosokawa K, Kido T, Kurata A, Okayama H, Higaki J, Mochizuki T, Murase K. Peri-infarct dysfunction in post-myocardial infarction: assessment of 3-T tagged and late enhancement MRI. Eur Radiol 2010;20:1139-48.

22. Koos R, Altiok E, Doetsch J, Neizel M, Krombach G, Marx N, Hoffmann R. Layer-specific strain-encoded MRI for the evaluation of left ventricular function and infarct transmurality in patients with chronic coronary artery

Cite this article as: Huo $\mathrm{H}$, Dai $\mathrm{X}$, Li S, Zheng $\mathrm{Y}$, Zhou J, Song Y, Liu S, Hou Y, Liu T. Diagnostic accuracy of cardiac magnetic resonance tissue tracking technology for differentiating between acute and chronic myocardial infarction. Quant Imaging Med Surg 2021;11(7):3070-3081. doi: 10.21037/qims-20-1109 disease. Int J Cardiol 2013;166:85-9.

23. Kihlberg J, Haraldsson H, Sigfridsson A, Ebbers T, Engvall JE. Clinical experience of strain imaging using DENSE for detecting infarcted cardiac segments. J Cardiovasc Magn Reson 2015;17:50.

24. Goto $Y$, Ishida $M$, Takase $S$, Sigfridsson A, Uno M, Nagata M, Ichikawa Y, Kitagawa K, Sakuma H. Comparison of displacement encoding with stimulated echoes to magnetic resonance feature tracking for the assessment of myocardial strain in patients with acute myocardial infarction. Am J Cardiol 2017;119:1542-7.

25. Maret E, Todt T, Brudin L, Nylander E, Swahn E, Ohlsson JL, Engvall JE. Functional measurements based on feature tracking of cine magnetic resonance images identify left ventricular segments with myocardial scar. Cardiovasc Ultrasound 2009;7:53.

26. Azarisman SM, Carbone A, Shirazi M, Bradley J, Teo KS, Worthley MI, Worthley SG. Characterisation of myocardial injury via T1 mapping in early reperfused myocardial infarction and its relationship with global and regional diastolic dysfunction. Heart Lung Circ 2016;25:1094-106.

27. Bachner-Hinenzon N, Malka A, Barac Y, Meerkin D, Ertracht O, Carasso S, Shofti R, Leitman M, Vered Z, Adam D, Binah O. Strain Analysis in the Detection of Myocardial Infarction at the Acute and Chronic Stages. Echocardiography 2016;33:450-8.

28. Liu T, Wang C, Li S, Zhao Y, Li P. Age- and genderrelated normal references of right ventricular strain values by tissue tracking cardiac magnetic resonance: results from a Chinese population. Quant Imaging Med Surg 2019;9:1441-50. 